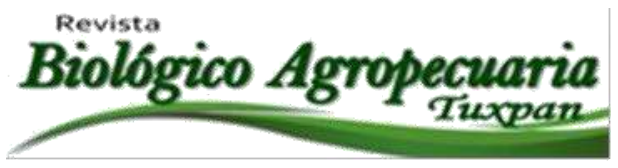

\title{
Comportamiento poblacional de Eisenia foetida y calidad fisicoquímica del humus en diferentes sustratos del trópico húmedo
}

Eisenia foetida population behavior and physico-chemical quality of humus in different substrates

$$
\text { humid tropics }
$$

Castro Málaga Verónica ${ }^{1}$, Alejandro Retureta Aponte ${ }^{2 \bowtie}$, Dinora Vázquez-Luna ${ }^{3}$ y María Gisela Velázquez Silvestre ${ }^{4}$

1,2,3,4 Facultad de Ingeniería en Sistemas de Producción Agropecuaria. Universidad Veracruzana, Km 22 carretera Costera del Golfo Acayucan-Catemaco, Acayucan, Veracruz. CP. 96000

${ }^{\square}$ Autor para correspondencia: aretureta@uv.mx

Recibido: $19 / 08 / 2016$

Aceptado: 22/11/2016

\section{RESUMEN}

Se evaluó el comportamiento poblacional de la lombriz Eisenia foetida (Adultas- huevos) así como la calidad fisicoquímica del humus, proveniente de tres sustratos (ovino, bovino, cachaza) y sus combinaciones, con el objetivo de determinar el sustrato con mayor calidad fisicoquímica de acuerdo con la norma NMX-FF-109-SCFI-2008. La mayor dinámica poblacional se encontró en el tratamiento combinado de ovino+bovino+cachaza. El pH con rango aceptable (8.4 y 8.3) se observó en ovino y bovino, las demás tratamientos mostraron rangos altos de alcalinidad, reflejados en la conductividad eléctrica. La Materia Orgánica de todos los tratamientos se localizó en un nivel admisible (29 a 33\%). El nitrógeno se mantuvo en niveles críticos (1.15 a 1.41\%), mientras que el fosforo, el hierro, el magnesio y el zinc se conservaron en niveles superiores. La concentración de potasio fue satisfactoria en los tratamientos de bovino, ovino+cachaza, bovino+cachaza, ovino+bovino+cachaza. Por el contrario, el cobre estuvo en niveles tóxicos en todos los tratamientos, con concentraciones mayores de $20 \mathrm{mg} \mathrm{kg}{ }^{-1}$. El manganeso solo se encontró en niveles aceptables en los tratamientos de ovino, ovino+cachaza, ovino+bovino+cachaza; ubicándose en un rango toxico en los demás tratamientos. Se consideró que el mejor sustrato de acuerdo con sus características físicoquímicas fue ovino+bovino+cachaza. Se considera pertinente investigar en el desarrollo de las plantas.

Palabras clave: Comportamiento, humus de lombriz, microelementos, toxicidad, trópico húmedo

\begin{abstract}
In this study, we evaluated the population behavior of Eisenia foetida worm, as well as the physicochemical quality of humus vermicompost from three substrates (ovine, bovine, cachaza) and
\end{abstract}


combinations thereof, in order to determine the substrate more physicochemical quality. The chemical characteristics of humus were determined according to the NMX-FF-109-SCFI-2008 standard. The best population dynamics (adults and eggs) was found was found in the combination of bovine + ovine + cachaza. The $\mathrm{pH}$ reported in the different substrates indicates that ovine and bovine treatments were found in an acceptable range, but not other combinations, which showed high alkalinity ranges reflected in the electrical conductivity. Organic Matter of all treatments was located at an acceptable level (29-33\%). Nitrogen remained at critical levels (1.15 to $1.41 \%$ ), while phosphorus, iron, magnesium and zinc were stored at higher levels. The concentration of potassium was successful in the treatment of bovine, ovine + cachaza, cachaza + bovine, ovine + bovine + cachaza. Conversely, copper was in toxic levels in all treatments, with higher concentrations $20 \mathrm{mg} \mathrm{kg}^{-1}$. Manganese was found only at acceptable levels in the treatment of ovine, ovine + cachaza, ovine + bovine + cachaza; It is located in a toxic range in the other treatments. Given the above, the best substrate in accordance with their physicochemical characteristics was the combination ovine + bovine + cachaza, although it is considered appropriate to investigate in later works, the development of plants.

Key words: California red worm, organic production, sustainability, microelements, toxicity

\section{INTRODUCCIÓN}

La lombricultura es una de las mejores técnicas que tiene la agricultura orgánica para mejorar la calidad de los suelos y ayudar a mantener beneficios integrales en lo agrícola, social y económico (Schuldt, 2006). En México, la aplicación de abonos orgánicos se remonta a nuestros ancestros, en la actualidad, el uso de Eisenia foetida se ha popularizado a nivel mundial por ser una lombriz domesticada, muy útil como herramienta de trabajo para reciclar la materia orgánica, obteniendo desde humus como resultado de la alimentación de la lombriz, hasta la industrialización de esta misma en la producción de carne y harina de lombriz, por lo que financieramente esta técnica es eficiente y rentable.

La factibilidad de estos sistemas también radica en la gran oferta de desechos orgánicos generadas a partir del desarrollo de las actividades productivas tanto urbanas como rurales, las cuales representan una fuente potencial de contaminación, siendo esta técnica una opción para dar valor agregado a éstos despedicios (Chávez-Porras et al., 2013), a fin de convertirlos en productos útiles (Bollo, 1999). El uso de la vermicomposta es muy variado y aunque puede usarse como mejorador del suelo, es necesario tener conocimiento de sus propiedades para no alterar de forma negativa la bioquímica del suelo. Durante el proceso de producción de vermicomposta intervienen varios factores tales como el tipo de sustrato, la especie, la densidad de lombrices, la humedad de la composta y las condiciones de las instalaciones utilizadas (K1z1lkaya \& Türkay, 2014), por lo que el objetivo del presente trabajo fue evaluar el comportamiento poblacional de la lombriz Eisenia foetida, así como la calidad fisicoquímica del humus de vermicomposta proveniente de tres sustratos (ovino, bovino, cachaza) y sus combinaciones,

Revista Científica Biológico Agropecuaria Tuxpan 4 (2) ISSN: 2007-6940 
con la finalidad de determinar el sustrato con mayor calidad fisicoquímica.

\section{MATERIALES Y MÉTODOS}

La investigación se realizó en el vivero de la Facultad de Ingeniería en Sistemas de Producción Agropecuaria (FISPA-UV), campus Acayucan, ubicado a $18^{\circ} 00^{\prime} 10.45^{\prime}, \quad \mathbf{N}$ y 9455'38.21', O. Se evaluaron siete tratamientos (Cuadro 1) con tres repeticiones,

\begin{tabular}{|c|c|c|c|}
\hline Tratamiento & Descripción & Identificación & Proporciones \\
\hline $\mathrm{T} 1$ & Estiércol de ovino & $\mathrm{EO}$ & Estiércol Ovino $100 \%$ \\
\hline $\mathrm{T} 2$ & Estiércol de bovino & $\mathrm{EB}$ & Estiércol Bovino 100\% \\
\hline $\mathrm{T} 3$ & Cachaza & $\mathrm{C}$ & Cachaza $100 \%$ \\
\hline $\mathrm{T} 4$ & $\begin{array}{l}\text { Estiércol de ovino + estiércol de } \\
\text { bovino }\end{array}$ & $\mathrm{EO}+\mathrm{EB}$ & $\begin{array}{l}\text { Estiércol Ovino } 50 \%+ \\
\text { estiércol Bovino } 50 \%\end{array}$ \\
\hline $\mathrm{T} 5$ & Estiércol de ovino + Cachaza & $\mathrm{EO}+\mathrm{C}$ & $\begin{array}{l}\text { Estiércol Ovino 50\%+ } \\
\text { Cachaza } 50 \%\end{array}$ \\
\hline T6 & Estiércol de bovino + Cachaza & $\mathrm{EB}+\mathrm{C}$ & $\begin{array}{l}\text { Estiércol Bovino } 50 \% \\
\text { +Cachaza } 50 \%\end{array}$ \\
\hline $\mathrm{T} 7$ & $\begin{array}{l}\text { Estiércol de ovino + estiércol de } \\
\text { bovino + Cachaza }\end{array}$ & $\mathrm{EO}+\mathrm{EB}+\mathrm{C}$ & $\begin{array}{l}\text { Estiércol Ovino } \\
\text { 33.3\%+estiércol Bovino } \\
\text { 33.3\%+Cachaza 333\% }\end{array}$ \\
\hline
\end{tabular}

Cuadro 1. Descripción de los tratamiento utilizados en el experimento Eisenia foetida (Savigny, 1826) en tres sustratos diferentes y sus combinaciones. mediante un diseño experimental completamente al azar, para ello se utilizaron 28 recipientes de plástico de $33 \mathrm{~cm}$ de largo $\mathrm{x}$ $21 \mathrm{~cm}$ de ancho x $12 \mathrm{~cm}$ de alto, los cuales fueron perforadas en la parte inferior, para drenar el exceso de agua. Cabe mencionar que previo a la preparación de las unidades experimentales, cada estiércol se precomposteó por diez días. 
experimental y se le añadieron 10 lombrices. El experimento tuvo una duración de 65 días, tiempo al que se realizó el conteo final. Durante el desarrollo del experimento se aplicaron riegos cada tercer día, manteniendo la humedad del suelo en 30\%. Para mantener una oxigenación adecuada se daba vuelta al sustrato cada semana, para que las lombrices pudieran tener acceso a todo el sustrato y no solamente se alimentaran en un solo lado.

Las variables de estudio fueron densidad poblacional de huevecillos, juveniles y adultas (Loh et al., 2005) y las propiedades del humus de lombriz (Materia Orgánica, Relación C/N, N, P, $\mathrm{K}, \mathrm{Ca}, \mathrm{Mg}, \mathrm{Fe}, \mathrm{Cu}, \mathrm{Zn}$ y $\mathrm{Mn}$ ). Las

características fisicoquímicas de la lombricomposta (Morales-Murguía et al., 2009) fueron determinadas de acuerdo con la norma NMX-FF-109-SCFI-2008.

Finalmente, los datos fueron analizados mediante un ANOVA mediante la prueba de Tukey $(\mathrm{p}<0.05)$ apoyado con el software para análisis estadístico SAS (Statistical Analysis System) versión 9.1 (SAS, 2004).

\section{RESULTADOS Y DISCUSIÓN}

Los resultados obtenidos después de 65 días de vermicomposteo evidenció la mayor

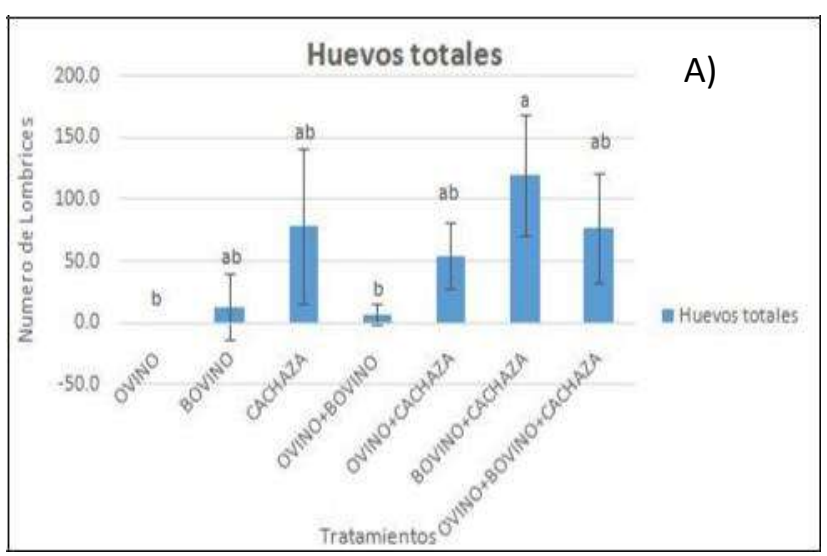

población de lombrices en el tratamiento de $\mathrm{EO}+\mathrm{EB}+\mathrm{C}$, mientras en la que la población se mantuvo baja en el de ovino (Figura 1). Al respeto, Pineda (2006) indica que el $\mathrm{pH}$ es determinante en el desarrollo poblacional de esta lombriz, por lo que el $\mathrm{pH}$ de 4.0 encontrado en el estiércol de ovino explicaría lo encontrado en este estudio. En relación con la población de lombrices juveniles no se encontraron diferencia significativas entre tratamientos ( $p>0.05)$, este se debió a la alta heterogeneidad encontrada (Figura 2). Esto pude deberse a la menor adaptación de las lombrices a condiciones de acidez, siendo a que por sus características de consistencia y compactación, debe ser abundantemente regado y mezclado a fondo con otros materiales como papel o paja. Se recomienda un precomposteo de 3 a 4 meses con riegos consecutivos, ya que en este trabajo no se le dio el tiempo indicado por Solano (2002). La cachaza es considerada como mejoradora de suelos, debido a sus propiedades amortiguadoras, siendo una alternativa en zonas cañeras o con producción mixta (ovino o bovino). Nuevamente, la mejor combinación fue $\mathrm{EO}+\mathrm{EB}+\mathrm{C}$, reflejado en la población adulta de $E$. foetida. Sin embargo, las combinaciones cachaza $\mathrm{EO}+\mathrm{C}$ y $\mathrm{EB}+\mathrm{C}$ pueden ser una alternativa para los productores que no que no cuenten con la anterior combinación.

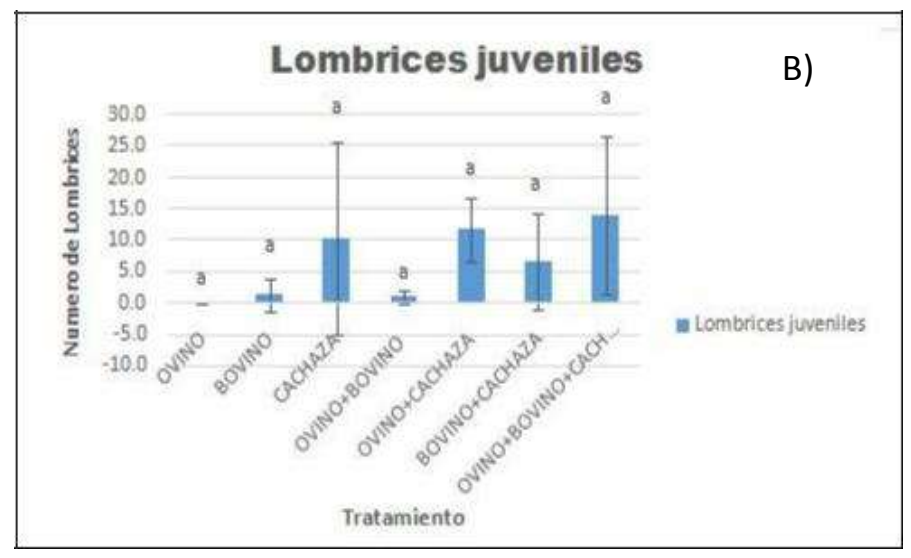


Figura 1. A) Población de huevos y B) lombrices juveniles de Eisenia foetida después de 65 días de vermicomposteo.
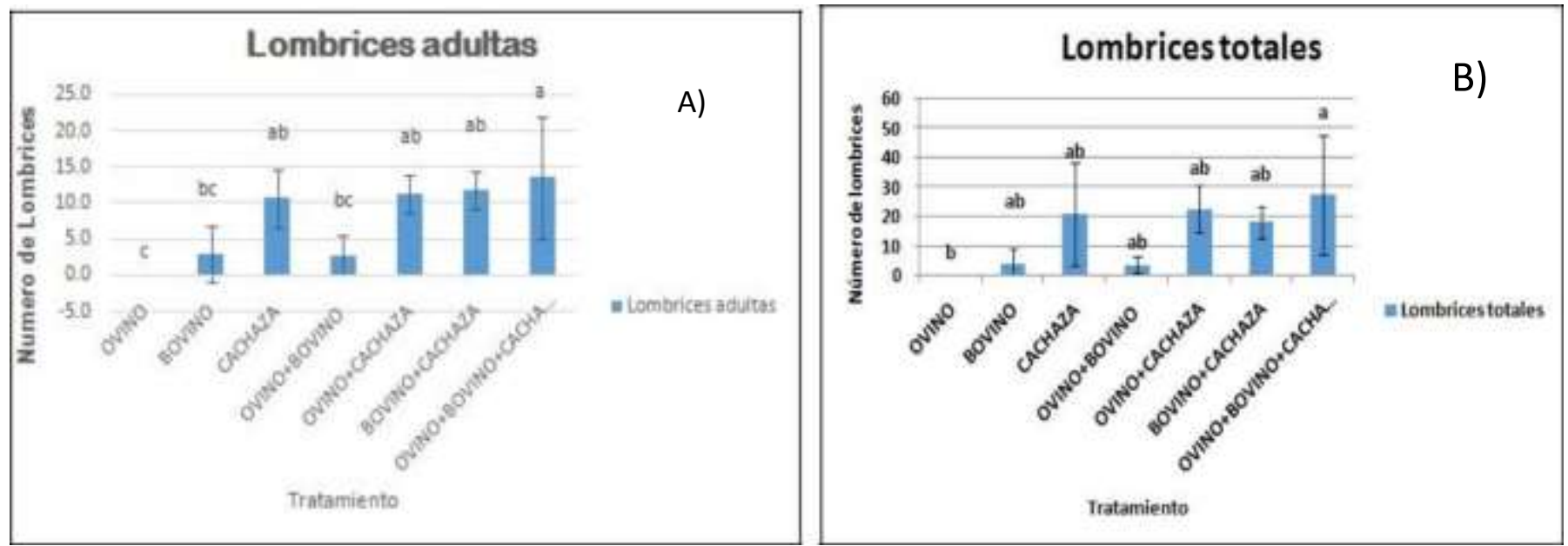

Figura 2. A) Población de lombrices adultas y B) lombrices totales de Eisenia foetida después de 65 días de vermicomposteo.

El pH reportado en los diferentes sustratos indica que los tratamientos ovino y bovino se encontraron en un rango aceptable, no así las demás combinaciones, las cuales mostraron rangos altos de alcalinidad, reflejados en la conductividad eléctrica. La Materia Orgánica de todos los tratamientos se localizó en un nivel admisible. El nitrógeno se mantuvo en niveles críticos, mientras que el fosforo, el hierro, el magnesio y el zinc se conservaron en niveles superiores. La concentración de potasio fue satisfactoria en los tratamientos de $\mathrm{EB}, \mathrm{EO}+\mathrm{C}, \mathrm{EB}+\mathrm{C}, \mathrm{EO}+\mathrm{EB}+\mathrm{C}$. Por el contrario, el cobre estuvo en niveles tóxicos en todos los tratamientos, con concentraciones mayores de $20 \mathrm{mg} \mathrm{kg}^{-1}$. El manganeso solo se encontró en niveles aceptables en los tratamientos de $\mathrm{EO}, \mathrm{EO}+\mathrm{C}$, $\mathrm{EO}+\mathrm{EB}+\mathrm{C}$; ubicándose en un rango toxico en los demás tratamientos.

Revista Científica Biológico Agropecuaria Tuxpan 4 (2)

ISSN: 2007-6940 
Cuadro 2. Propiedades químicas analizadas en la vermicomposta después de 65 días de composteo.

\begin{tabular}{ccccccccc}
\hline PROPIEDAD & & OVINO & BOVINO & CACHAZA & $\begin{array}{l}\text { OVINO+ } \\
\text { BOVINO }\end{array}$ & $\begin{array}{l}\text { OVINO+ } \\
\text { CACHAZA }\end{array}$ & $\begin{array}{l}\text { BOVINO+ } \\
\text { CACHAZA }\end{array}$ & $\begin{array}{l}\text { OVINO+ } \\
\text { BOVINO+ } \\
\text { CACHAZA }\end{array}$ \\
\hline $\mathbf{p H}$ & & 8.42 & 8.36 & 9.21 & 8.72 & 9.17 & 8.93 & 9.04 \\
$\begin{array}{c}\text { Conductividad } \\
\text { Eléctrica }\end{array}$ & $\mathrm{ds} \mathrm{m}^{-1}$ & 3.01 & 2.18 & 2.47 & 3.46 & 4.01 & 4.16 & 3.46 \\
\hline Materia Orgánica & & 33.85 & 34.19 & 28.92 & 31.67 & 30.25 & 32.11 & 29.56 \\
$\mathbf{C} / \mathbf{N}$ & & 15.67 & 16.75 & 16.14 & 14.93 & 14.16 & 16.06 & 15.11 \\
$\mathbf{N}$ & & 1.26 & 1.23 & 1.24 & 1.31 & 1.41 & 1.32 & 1.15 \\
$\mathbf{P}$ & $\%$ & 5.26 & 4.22 & 5.71 & 6.06 & 5.17 & 5.09 & 4.27 \\
$\mathbf{K}$ & & 0.41 & 1.05 & 0.86 & 0.64 & 1.07 & 1.11 & 1.17 \\
$\mathbf{C a}$ & & 5.81 & 4.03 & 4.12 & 5.72 & 4.24 & 5.06 & 3.97 \\
$\mathbf{M g}$ & & 0.86 & 1.02 & 1.04 & 0.99 & 1.01 & 1.12 & 1.08 \\
\hline $\mathbf{F e}$ & & 66.81 & 93.6 & 73.74 & 74.82 & 76.68 & 65.43 & 85.4 \\
$\mathbf{C u}$ & & 33.15 & 24.18 & 39.25 & 26.32 & 31.72 & 27.33 & 24.71 \\
$\mathbf{Z n}$ & $\mathrm{mg.kg}{ }^{-1}$ & 44.83 & 32.74 & 49.08 & 48.14 & 45.22 & 33.96 & 31.19 \\
$\mathbf{M n}$ & & 320.75 & 291.6 & 373.09 & 356.45 & 287.44 & 318.7 & 286.03 \\
\hline
\end{tabular}

\section{CONCLUSIÓN}

En este estudio la mejor dinámica poblacional (adultas y huevos) fue hallada en la combinación de $\mathrm{EO}+\mathrm{EB}+\mathrm{C}$. En cuanto a la calidad del humus, se encontraron parámetros críticos $(\mathrm{pH}, \mathrm{N}$ y la relación $\mathrm{C} / \mathrm{N})$ para algunos sustratos. La concentración de cobre estuvo en niveles tóxicos en todos los tratamientos, con concentraciones mayores de $20 \mathrm{mg} \mathrm{kg}^{-1}$. El manganeso solo se encontró en niveles aceptables en $\mathrm{EO}, \mathrm{EO}+\mathrm{C}, \mathrm{EO}+\mathrm{EB}+\mathrm{C}$; ubicándose en un rango toxico en los demás tratamientos. Por todo lo anterior, el mejor sustrato de acuerdo con sus características químicas fue la combinación $\mathrm{EO}+\mathrm{EB}+\mathrm{C}$, aunque se considera pertinente investigar en trabajos posteriores, el desarrollo de las plantas.

\section{LITERATURA CITADA}

Bollo, E. (1999). Lombricultura: Una alternativa de reciclaje. Quito.: SobocGrafic.

Chávez-Porras, Á., Rodríguez-González, A., \& González-Gómez, F. E. (2013). Análisi de la dinámica biológica presente en un sistema piloto de lombricultura para el manejo de biosólidos provenientes de aguas residuales. Revista Ingenierías Universidad de Medellín, 12(22), 13-24. , 13-24.

https://doi.org/10.22395/rium.v12n22a1 35 
K1zılkaya, R., \& Türkay, F. (2014).

Vermicomposting of anaerobically digested sewage sludge with hazelnut husk and cow manure by earthworm Eiseniafoetida.Compost Science \& Utilization , 68-82.

https://doi.org/10.1080/1065657X.2014.8

Loh, T. H., Lee, Y. C., Liang, J. B., \& Tan, D. (2005). Vermicomposting of cattle and goat manures by Eisenia fetida and their growth and reproduction performance.

Bioresourse technology, 111-114.

https://doi.org/10.1016/j.biortech.2003.03

Morales-Murguía, J., Fernández, R., Montiel, C. A., \& Peralta, B. (2009). Evaluación de sustratos orgánicos en la producción de lombricomposta y el desarrollo de la lombriz (Eisenia foetida). Biotecnia , 19-26. https://doi.org/10.18633/bt.v11i1.49

Pineda, J. A. (2006). Lombricultura. Tegucigalpa, Honduras: Instituto Hondureño del Café.

SAS. (2004). SAS/GRAPH 9.1 Reference. North Carolina, USA: SAS Institute.

Schuldt, M. (2006). Lombricultura: Teoría y práctica. Mundi-Prensa.

Solano, F. V. (2002). Manual agropecuario, tecnologías orgánicas de la granja integral autosuficiente. Colombia: Ibalpe. 
Copyright (c) 2016 Verónica Castro Málaga, Alejandro Retureta Aponte,

Dinora Vázquez Luna y Maria Gisela V elázquez Silvestre

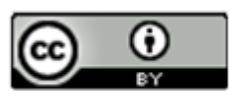

Este tex to está protegido por una licencia licencia Creative Commons 4.0

Usted es libre para Compartir —copiar y redistribuir el material en cualquier medio o form ato- y Adaptar el documento —remezclar, transformar y crear a partir del material- para cualquier propósito, , incluso para fines comerciales, siempre que cumpla 1a condición de:

\begin{abstract}
Atribución: Usted debe dar crédito a la obra original de manera adecuada, proporcionar un enlace a la licencia, e in dicar si se han realizado cam bios. Puede hacerlo en cualquier form a razonable, pero no de form a tal que sugiera que tiene el apoyo del licenciante o 10 recibe por el uso que hace de la obra.
\end{abstract}

Resumendelicencia - Textocompletodelalicencia 